\title{
Molecular Modelling and Evaluation of Hidden Information in ABCB11 Gene Mutations
}

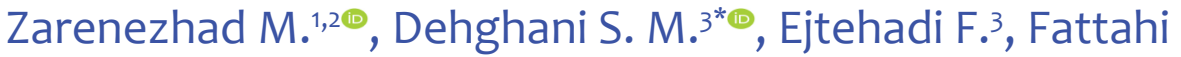 \\ M. R. ${ }^{3}$, Mortazavi M. ${ }^{4}$, Tabei S. M. B. ${ }^{5}$
}

\begin{abstract}
Background: Cholestatic disorders are divided in the extra and intra-hepatic that created due to the severe liver diseases. ABCB11 encodes the bile salt export pump and this gene is mutated in several forms of intrahepatic cholestasis. So far, some molecular features of this gene was studies.

Objective: Using a developed web server, we identified high number of rare codons in this gene, and four cases were related to BSEP-deficient patients which can be used for drug design.
\end{abstract}

Material and Methods: By in-silico modelling of $\mathrm{ABCB} 11$, some of rare codons in different locations of ATP8b1 gene were identified and evaluated. Using several web servers a number of mutations that converted non-rare codons to rare codon in these patients were identified.

Results: Some of these rare Codons were located at special positions by mutation of which, the new side chains do not seem suitable for protein structure and function. Furthermore, this mutation changed the protein folding rate that may have a critical role in proper folding. Thus, primary change of these codons contributes to BSEP deficiency.

Conclusion: This work is a comprehensive analysis of rare codons of ABCB11 and assessment of a number of these rare codon in protein levels. Rare codons evaluation can enhance our understanding of $\mathrm{ABCB} 11$ structural protein of $\mathrm{ABCB} 11$, and help us to develop mutation-specific therapies in design of new drugs.

Citation: Zarenezhad M, Dehghani S. M, Ejtehadi F, Fattahi M. R, Mortazavi M, Tabei S. M. B. Evaluation of Hidden Information in ABCB11 Gene Mutations. J Biomed Phys Eng. 2019;9(3):303-316. https://doi.org/10.31661/jbpe.v9i3Jun.680.

Keywords

ABCB11, Bioinformatics Analysis, Rare Codon, Mutation

\section{Introduction}

$\mathrm{T}$ he bile salt export pump (BSEP, protein product of the ABCB11 gene) is situated in the canalicular membrane of hepatocytes and is responsible for the translocation of bile salts [1]. BSEP belongs to $\mathrm{ABC}$ transporter superfamily and has 12 -transmembrane span integral membrane proteins. Mutations in ABCB11 are related to a phenotypical spectrum of cholestatic liver diseases. Cholestasis is created due to severe liver diseases where bile cannot flow from the liver to the duodenum [2]. The causes of cholestasis are divided into two groups: those originating outside the liver and those originating within the liver [3]. Some reasons of the cholestasis within the liver include cirrhosis due to viral hepatitis B or C, drugs, acute hepatitis, alcoholic liver dis-

${ }^{1} \mathrm{MD}, \mathrm{PhD}$, Gastroenterohepatology Research Center, Shiraz University of Medical Sciences,

Shiraz, Iran

${ }^{2} \mathrm{MD}, \mathrm{PhD}$, legal medi-

cine research center, legal medicine organiza-

tion, Tehran , iran

${ }^{3} \mathrm{MD}$, Gastroentero-

hepatology Research

Center, Shiraz University

of Medical Sciences,

Shiraz, Iran

${ }^{4} \mathrm{PhD}$, Department of

Biotechnology, Institute

of Science and High

Technology and Envi-

ronmental Science,

Graduate University of

Advanced Technology,

Kerman, Iran

${ }^{5} \mathrm{MD}$, Genetic Research

Center, Shiraz University

of Medical Sciences,

Shiraz, Iran

*Corresponding author:

S. M. Dehghani, MD

Gastroenterohepatology Research Center, Shiraz University of Medical Sciences, Shiraz, Iran E-mail: dehghanism@ gmail.com

Received: 1 November 2016 Accepted: 20 December 2016 
ease, primary biliary cirrhosis with inflammation and scarring of the bile ducts [4]. At present, specific gene defects have been identified for PFIC2 which are caused by the deficient in gene product required for bile formation and canalicular export $[2,5]$. Previously, the molecular evaluation of these PFICs including exon characterization [6], locus mapping [7], sequencing [8] and gene mutations have been studied.

The rare codons that are introduced as genetic hidden information are shown to have a critical role in protein activity and folding, and can help in problem solving of diseases and drug design $[9,10]$. Codon-usage analysis can also contribute to understanding the interaction between RNA viruses and the immune response of the hosts [10]. Several mutations of ABCB11 associated with BSEP deficiency have previously been introduced $[1,11]$. However, there is no study about rare codons of ABCB11 gene, and in this study we evaluated the situation of these rare codons and variations in the structure of ABCB11. For this, by submission of PFIC3 gene in the I-TASSER server, a three dimensional model of BSEP protein was created [12]. In addition, rare codons of ABCB11 gene were detected using the Sherlocc program [13], LaTcOm (http://structure.biol.ucy.ac.cy/latcom.html) [14], ATGme [15] and RaCC server (http://nihserver.mbi. ucla.edu/RACC/). By PyMOL [16] and SPDBV software [17], the characteristics of these rare codons were studied in the 3D model of BSEP protein. In the following, these variations and their relationships with rare codon, were evaluated. In this study, a large number of genetic mutations of ABCB11gene were evaluated for their relations with rare codons and PFIC2 disease. Furthermore, some interesting results demonstrate that some of these mutations have a destructive effect on the structure of BSEP protein and result in PFIC2 disease. These findings help the elucidation of hidden information of this gene. The overall results of this study are thought to be useful in the design of new efficient drugs.

\section{Material and Methods}

\section{Rare Codons Analysis}

For bioinformatics analysis of ABCB11 gene, the nucleotide sequences and features of this gene were retrieved from http://www. ncbi.nlm.nih.gov/genome/. By use of nucleotide sequence of ABCB11 gene, rare codons of this gene were detected using the following servers. Rare codon calculator (RaCC) (http:// nihserver.mbi.ucla.edu/RACC/) detected problematic residues as arginine (AGG, AGA, CGA), leucine (CTA), isoleucine (ATA) and proline (CCC). ATGme [15] detected rare codon in three steps: (i) Input of the ATP8b1 sequence; (ii) Input of the codon usage table of Homo sapiens [gbpri]: 93487 CDS's (iii) Detection of rare codons. LaTcOm [14] reported three algorithms are implemented for the detection of rare codon clusters: i) \% minimax algorithm, ii) sliding window approach and iii) a linear-time algorithm named MSS. Then, the RCC positions were visualized within the submitted sequences. Sherlocc's program [13] detected rare codon clusters by retrieving the nucleotide sequence of proteins in each Pfam protein family alignments. By these servers, some rare codons were identified in the nucleotide sequences of ABCB11 gene.

\section{Study of Rare Codons in the Struc-} ture of $A B C B 11$

To evaluate the position of these new identified mutations and rare codon in the structure of BSEP protein, a 3D structure of BSEP was created in the I-TASSER web server [12] based on multiple-threading alignments by LOMETS [18]. Models with the best "Confidence Score" and Z-score were chosen by I-TASSER server. The best model with suitable Z-score and confidence was visualized using PyMOL [16] and Swiss PDB viewer [17]. With Expasy's Prot Param (http://us.expasy.org/tools/ protparam. html) server, the total number of positive and 
negative residues, physico-chemical parameters, molecular weight and other features of this model were computed. Hydrogen bonds were also detected by PIC web server [19] and WHAT IF web server [20]. Finally, the situation and relationships of these mutations and rare codons were evaluated in the structure of BSEP.

\section{Results}

Preparation of Molecular Modelling Structure of ABCB11

For understanding the protein structure and function, preparation of 3D structure of protein is a vital process in biology [21]. Our studies show that heretofore has not been determined as the crystal structure of BSEP, and it is obligatory to provide the 3D model from this protein. For this, by submitting the sequence of ATP8B1 in I-TSSAR Web Server, 3D models of these proteins were obtained. The I-TSSAR Web Server generated five models and best model showed -1.82 values of overall C-score, $0.49 \pm 0.15$ value of TM-Score and Exp. RMSD was 14.1 \pm 3.9 (Figure 1).

In the following, the physiochemical proper-

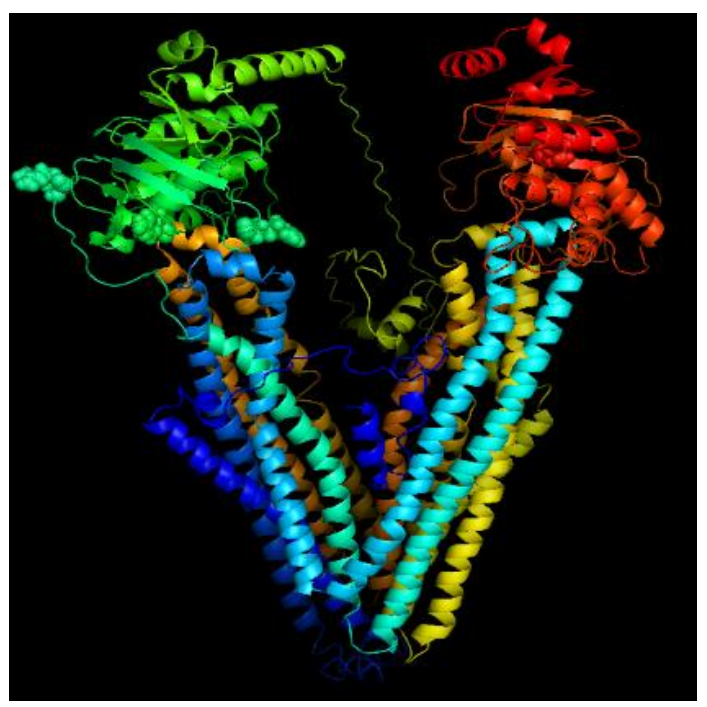

Figure 1: The ribbon diagram of BSEP protein modelled in I-TSSAR Web Servers. ties of ATP8B1 protein model were calculated in ProtParam tool (Table 1) [22].

\section{Detection of Rare Codon Clusters}

Using UniProt database (http://www.uniprot. org/), the Pfam accession number of BSEP protein was identified as PF00664 (ABC_ membrane. 2 hits) and PF00005 (ABC_tran. 2 hits). Pfam is a comprehensive collection of protein domains and families [23]. These Pfam was analyzed in the Sherlocc program [13] and results show that any rare codon cluster was identified in these Pfam accession number of ABCB11 genes. Next, the nucleotide sequence of $\mathrm{ABCB} 11$ gene was analyzed in ATGme server [15]. Using the codon usage table of Homo sapiens [gbpri]: 93487 CDS's (40662582 codons) (http://www.kazusa.or.jp/ codon/cgi-bin/showcodon.cgi?species $=9606$ ), this gene was analyzed, and the rare and highly rare codons were shown and highlighted in orange and red, respectively (Figure 2). Moreover, GC and AT contents of this gene were GC\%: 45.44, AT\%:54.56, calculated by this server.

In the following, RaCC server was used. By introduction of problematic residue codons as

Table 1: In silico physico-chemical features of BSEP protein obtained from ProtParam tool.

\begin{tabular}{lc} 
Parameters & ATP8b1 \\
\hline Theoretical pl & 6.17 \\
\hline Molecular weight & 146407.18 \\
\hline Sequence length & 1321 \\
\hline Extinction coefficients (M-1 cm- & $129370-128120$ \\
1at 260 nm) & 143 \\
\hline Asp + Glu & 135 \\
\hline Arg + Lys & 33.29 \\
\hline Instability index & 0.011 \\
\hline Grand average of hydropathicity & 92.60
\end{tabular}

${ }^{*}$ First number is based on the assumption that both cysteine residues form cystine and the second number that both cysteines are reduced. 


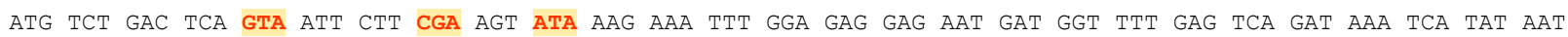
AAT GAT AAG AAA TCA AGG TTA CAA GAT GAG AAg AAA GGT GAT GGC GTT AgA GTT GGC TTC TTT CAA TTG TTT CGG TTT TCT TCA TCA ACT GAC ATT TGG CTG ATG TTT GTG GGA AGT TTG TGT GCA TTT CTC CAT GGA ATA GCC CAG CCA GGC GTG CTA CTC ATT TTT GGC ACA ATG ACA GAT GTT TTT ATT GAC TAC GAC GTT GAG TTA CAA GAA CTC CAG ATT CCA GGA AAA GCA TGT GTG AAT AAC ACC ATT GTA TGG ACT AAC AGT TCC CTC AAC CAG AAC ATG ACA AAT GGA ACA CGT TGT GGG TTG CTG AAC ATC GAG AGC GAA ATG ATC AAA TTT GCC AGT TAC TAT GCT GGA ATT GCT GTC GCA GTA CTT ATC ACA GGA TAT ATT CAA ATA TGC TTT TGG GTC ATT GCC GCA GCT CGT CAG ATA CAG AAA ATG AGA AAA TTT TAC TTT AGg AGA ATA ATG AGA ATG GAA ATA GGG TGG TTT GAC TGC AAT TCA GTG GGG GAG CTG AAT ACA AGA TTC TCT GAT GAT ATT AAT AAA ATC AAT GAT GCC ATA GCT GAC CAA ATG GCC CTT TTC ATT CAG CGC ATG ACC TCG ACC ATC TGT GGT TTC CTG TTG GGA TTT TTC AGg GGT TGG AAA CTG ACC TTG GTT ATT ATT TCT GTC AGC CCT CTC ATT GGG ATT GGA GCA GCC ACC ATt GGT CTG AGT GTG TCC AAG TTT ACG GAC TAT GAG CTG AAG GCC TAT GCC AAA GCA GGG GTG GTG GCT GAT GAA GTC ATT TCA TCA ATG AGA ACA GTG GCT GCT TTT GGT GGT GAG AAA AGA GAG GTT GAA AGg TAT GAg AAA AAT CTT GTG TTC GCC CAG CGT TGG GGA ATT AGA AAA GGA ATA GTG ATG GGA TTC TTT ACT GGA TTC GTG TGG TGT CTC ATC TTT TTG TGT TAT GCA CTG GCC TTC TGG TAC GGC TCC ACA CTT GTC CTG GAT GAA GGA GAA TAT ACA CCA GGA ACC CTT GTC CAG ATT TTC CTC AGT GTC ATA GTA GGA GCT TTA AAT CTT GGC AAT GCC TCT CCT TGT TTG GAA GCC TTT GCA ACT GGA CGT GCA GCA GCC ACC AGC ATT TTT GAG ACA ATA GAC AGG AAA CCC ATC ATT GAC TGC ATG TCA GAA GAT GGT TAC AAG TTG GAT CGA ATC AAG GGT GAA ATT GAA TTC CAT AAT GTG ACC TTC CAT TAT CCT TCC AGA CCA GAG GTG AAG ATT CTA AAT GAC CTC AAC ATG GTC ATT AAA CCA GGg GAA ATG ACA GCT CTG GTA GGA CCC AGT GGA GCT GGA AAA AGT ACA GCA CTG CAA CTC ATT CAG CGA TTC TAT GAC CCC TGT GAA GGA ATG GTG ACC GTG GAT GGC CAT GAC ATT CGC TCT CTT AAC ATT CAG TGG CTT AGA GAT CAG ATT GGG ATA GTG GAg CAA GAg CCA GTT CTG TTC TCT ACC ACC ATT GCA GAA AAT ATT CGC TAT GGC AGA GAA GAT GCA ACA ATG GAA GAC ATA GTC CAA GCT GCC AAG GAG GCC AAT GCC TAC AAC TTC ATC ATG GAC CTG CCA CAG CAA TTT GAC ACC CTT GTT GGA GAA GGA GGA GGC CAG ATG AGT GGT GGC CAG AAA CAA AGG GTA GCT ATC GCC AGA GCC CTC ATC CGA AAT CCC AAG ATT CTG CTT TTG GAC ATG GCC ACC TCA GCT CTG GAC AAT GAG AGT GAA GCC ATG GTG CAA GAA GTG CTG AGT AAG ATT CAG CAT GGG CAC ACA ATC ATT TCA GTT GCT CAT CGC TTG TCT ACG GTC AGA GCT GCA GAT ACC ATC ATT GGT TTT GAA CAT GGC ACT GCA GTG GAA AGA GGG ACC CAT GAA GAA TTA CTG GAA AGG AAA GGT GTT TAC TTC ACT CTA GTG ACT TTG CAA AGC CAG GGA AAT CAA GCT CTT AAT GAA GAG GAC ATA AAG GAT GCA ACT GAA GAT GAC ATG CTT GCG AGG ACC TTT AGC AGA GGG AGC TAC CAG GAT AGT TTA AGG GCT TCC ATC CGG CAA CGC TCC AAG TCT CAG CTT TCT TAC CTG GTG CAC GAA CCT CCA TTA GCT GTT GTA GAT CAT AAG TCT ACC TAT GAA GAA GAT AGA AAG GAC AAG GAC ATt CCT GTG CAG GAA GAA GTT GAA CCT GCC CCA GTT AGg AGG ATt CTG AAA TTC AGT GCT CCA GAA TGG CCC TAC ATG CTG GTA GGG TCT GTG GGT GCA GCT GTG AAC GGG ACA GTC ACA CCC TTG TAT GCC TTT TTA TTC AGC CAG ATT CTT GGG ACT TTT TCA ATT CCT GAT AAA GAG GAA CAA AGG TCA CAG ATC AAT GGT GTG TGC CTA CTT TTT GTA GCA ATG GGC TGT GTA TCT CTT TTC ACC CAA TTT CTA CAG GGA TAT GCC TTT GCT AAA TCT GGG GAG CTC CTA ACA AAA AGG CTA CGT AAA TTT GGT TTC AGG GCA ATG CTG GGG CAA GAT ATT GCC TGG TTT GAT GAC CTC AGA AAT AGC CCT GGA GCA TTG ACA ACA AGA CTT GCT ACA GAT GCT TCC CAA GTT CAA GGG GCT GCC GGC TCT CAG ATC GGG ATG ATA GTC AAT TCC TTC ACT AAC GTC ACT GTG GCC ATG ATC ATT GCC TTC TCC TTT AGC TGG AAg CTG AGC CTG GTC ATC TTG TGC TTC TTC CCC TTC TTG GCT TTA TCA GGA GCC ACA CAG ACC AgG ATG TTG ACA GGA TTT GCC TCT CGA GAT AAg CAG GCC CTG GAg ATg GTG GGA CAG ATT ACA AAT GAA GCC CTC AGT AAC ATC CGC ACT GTT GCT GGA ATT GGA AAG GAG AGG CGG TTC ATT GAA GCA CTT GAG ACT GAG CTG GAG AAG CCC TTC AAG ACA GCC ATT CAG AAA GCC AAT ATT TAC GGA TTC TGC TTT GCC TTT GCC CAG TGC ATC ATG TTT ATT GCG AAT TCT GCT TCC TAC AGA TAT GGA GGT TAC TTA ATC TCC AAT GAG GGG CTC CAT TTC AGC TAT GTG TTC AGG GTG ATC TCT GCA GTT GTA CTG AGT GCA ACA GCT CTT GGA AGA GCC TTC TCT TAC ACC CCA AGT TAT GCA AAA GCT AAA ATA TCA GCT GCA CGC TTT TTT CAA CTG CTG GAC CGA CAA CCC CCA ATC AGT GTA TAC AAT ACT GCA GGT GAA AAA TGG GAC AAC TTC CAG GGG AAG ATT GAT TTT GTT GAT TGT AAA TTT ACA TAT CCT TCT CGA CCT GAC TCG CAA GTT CTG AAT GGT CTC TCA GTG TCG ATT AGT CCA GGG CAG ACA CTG GCG TTT GTT GGG AGC AGT GGA TGT GGC AAA AGC ACT AGC ATT CAG CTG TTG GAA CGT TTC TAT GAT CCT GAT CAA GGG AAG GTG ATG ATA GAT GGT CAT GAC AGC AAA AAA GTA AAT GTC CAG TTC CTC CGC TCA AAC ATT GGA ATT GTT TCC CAG GAA CCA GTG TTG TTT GCC TGT AGC ATA ATG GAC AAT ATC AAG TAT GGA GAC AAC ACC AAA GAA ATT CCC ATG GAA AGA GTC ATA GCA GCT GCA AAA CAG GCT CAG CTG CAT GAT TTT GTC ATG TCA CTC CCA GAG AAA TAT GAA ACT AAC GTT GGG TCC CAG GGG TCT CAA CTC TCT AGA GGG GAG AAA CAA CGC ATT GCT ATT GCT CGG GCC ATT GTA CGA GAT CCT AAA ATC TTG CTA CTA GAT GAA GCC ACT TCT GCC TTA GAC ACA GAA AGT GAA AAG ACG GTG CAG GTT GCT CTA GAC AAA GCC AGA GAG GGT CGG ACC TGC ATT GTC ATT GCC CAT CGC TTG TCC ACC ATC CAG AAC GCG GAT ATC ATT GCT GTC ATG GCA CAG GGG GTG GTG ATT GAA AAG GGG ACC CAT GAA GAA CTG ATG GCC CAA AAA GGA GCC TAC TAC AAA CTA GTC ACC ACT GGA TCC CCC ATC AGT TGA

Figure 2: Schematic representation of the rare (orange) and highly rare (red) codons in ABCB11gene.

Pro, Ile, Arg and Leu, this result was refined. This analysis shows that ABCB11 gene has 48 rare codons for Arg, 18 rare codons for Ile, 11 single rare codons for Leu and 11 rare codons for Pro (Figure 3). This analysis also showed that ABCB11 gene has two tandem double repeats of rare Arg codon.

Later, by LaTcOm web tool rare codon clusters of this gene were detected [14]. In LaTcOm, three algorithms of MSS, sliding window and \% MINMAX were employed. The reference codon usage table was used from CUTG database [24] these algorithms. Figure 4 shows the location of RCCs in this gene us- ing these algorithms (Figure 4; A, B and C).

These results demonstrate that MSS detected 6 clusters, Minmax detected 15 and slidingwindow detected 10 clusters. It is important to note that the cluster length selected for MSS algorithms was 21 codons and for Minmax and sliding-window algorithms were 25 codons. The characteristics and position of these RCCs in the ABCB11 gene were calculated (data not shown).

Evaluation of some Mutation Associated with Rare Codons

Some mutations in ABCB11 gene which 
atg tct gac tca gta att ctt $\underline{\text { CGA }}$ agt $\underline{\text { ATA }}$ aag aaa ttt gga gag gag aat gat ggt ttt gag tca gat aaa tca tat aat aat gat aag aaa tca AGG tta $\overline{c a a}$ gat $\overline{\text { gag }}$ aag aaa ggt gat ggc gtt AGA gtt ggc ttc ttt caa ttg ttt cgg ttt tct tca tca act gac att $\overline{\operatorname{tgg}}$ ctg atg ttt gtg gga agt ttg tgt gca ttt $\overline{c t c}$ cat gga ATA gcc cag cca ggc gtg CTA ctc att ttt ggc aca atg aca gat gtt ttt att gac tac gac gtt gag tta caa gaa ctc $\overline{c a g}$ att cca gga aaa gca tgt gtg aat aac acc att gta tgg act aac agt tcc ctc aac cag aac atg aca aat gga aca cgt tgt ggg ttg ctg aac atc gag agc gaa atg atc aaa ttt gcc agt tac tat get gga att gct gtc gca gta ctt atc aca gga tat att caa ATA tgc ttt tgg gtc att gcc gca get cgt cag ATA cag aaa atg AGA aaa ttt tac ttt AGG AGA ATA atg AGA atg gaa ATA ggg tgg ttt gac tgc aat tca gtg ggg gag $\overline{\mathrm{ctg}}$ aat aca AGA $\overline{\text { ttc }}$ tct gat gat att $\overline{\text { aat }} \overline{\text { aaa }} \overline{\text { atc }}$ aat gat gcc $\overline{\text { ATA }} \overline{\text { gct gac caa }}$ atg gcc ctt ttc att cag cgc atg acc tcg acc atc tgt ggt ttc ctg ttg gga ttt ttc AGG ggt tgg aaa ctg acc ttg gtt att att tct gtc agc cct ctc att ggg att gga gca gcc acc att ggt ctg agt gtg tcc aag ttt acg gac tat gag ctg aag gcc tat gcc aaa gca ggg gtg gtg gct gat gaa gtc att tca tca atg AGA aca gtg gct gct ttt ggt ggt gag aaa AGA gag gtt gaa AGG tat gag aaa aat ctt gtg ttc gcc cag cgt tgg gga att $\overline{\text { AGA }}$ aaa gga ATA gtg atg gga ttc ttt act gga ttc gtg $\overline{\operatorname{tgg}}$ tgt ctc atc ttt ttg tgt tat gca ctg gcc ttc tgg tac ggc tcc aca $\overline{c t t}$ gtc ctg gat gaa gga gaa tat aca cca gga acc ctt gtc cag att ttc ctc agt gtc ATA gta gga gct tta aat ctt ggc aat gcc tct cct tgt ttg gaa gcc ttt gca act gga cgt gca gca gcc acc agc att ttt gag aca ATA gac AGG aaa CCC atc att gac tgc atg tca gaa gat ggt tac aag ttg gat $\underline{C G A}$ atc aag ggt gaa att gaa ttc cat aat gtg acc ttc cat tat cct tcc AGA cca gag gtg aag att $\underline{\mathrm{CTA}}$ aat gac ctc $\overline{\mathrm{aac}}$ atg gtc att aaa cca ggg gaa atg aca gct ctg gta gga ccc agt gga gct gga aaa agt aca gca ctg caa ctc att cag CGA ttc tat gac CCC tgt gaa gga atg gtg acc gtg gat ggc cat gac att cgc tct ctt aac att cag tgg ctt AGA gat $\overline{c a g}$ att ggg ATA $\overline{\text { gtg }}$ gag caa gag cca gtt ctg ttc tct acc acc att gca gaa aat att cgc tat ggc AGA gaa gat gca aca atg gaa gac ATA gtc caa gct gcc aag gag gcc aat gcc tac aac ttc atc atg gac ctg cca cag caa ttt gac acc ctt gtt gga gaa gga gga ggc cag atg agt ggt ggc cag aaa caa AGG gta get atc gCC AGA gCC ctc atc CGA aat CCC aag att ctg ctt ttg gac atg gcc acc tca gct ctg gac aat gag agt gaa gcc atg gtg $\overline{c a a}$ gaa gtg ctg $\overline{\text { agt }}$ aag att cag cat ggg cac aca atc att tca gtt gct cat cgc ttg tct acg gtc AGA gct gca gat acc atc att ggt ttt gaa cat ggc act gca gtg gaa AGA ggg acc cat gaa gaa tta ctg gaa AGG aaa ggt gtt tac ttc act CTA gtg act ttg caa agc cag gga aat caa get $\overline{c t t}$ aat gaa gag gac ATA aag gat gca act gaa gat gac atg ctt gcg $\overline{A G G}$ acc ttt agc AGA ggg agc tac cag gat agt tta $A G G$ gct tcc atc $\overline{c g g}$ caa cgc tcc aag tct cag ctt tct tac ctg $\overline{g t g}$ cac gaa cct $\overline{c c a}$ tta get gtt gta gat cat aag tct acc tat gaa gaa gat AGA aag gac aag gac att cct gtg cag gaa gaa gtt gaa cct gcc cca gtt AGG AGG att ctg aaa ttc agt gct cca gaa tgg ccc tac atg ctg gta ggg tct gtg ggt gca gct gtg aac ggg aca gtc aca ccc ttg tat gcc ttt tta ttc agc cag att ctt ggg act ttt tca att cct gat aaa gag gaa caa AGG tca cag atc aat ggt gtg tgc CTA ctt ttt gta gca atg ggc tgt gta tct ctt ttc acC caa ttt CTA cag gga tat $\overline{\text { gCC }}$ ttt gct aaa tct ggg gag ctc $\overline{\text { CTA }}$ aca aaa AGG CTA cgt aaa ttt ggt ttc $\underline{\text { AGG }}$ gca atg ctg ggg $\overline{c a a}$ gat att gcc tgg ttt gat gac ctc $\overline{A G A}$ aat agc $\overline{c c t}$ gga gca $\overline{\text { ttg }} \overline{\text { aca }}$ aca $\overline{A G A}$ ctt gct aca gat gct tcc caa gtt caa ggg gct gcc ggc tct cag atc ggg atg ATA gtc aat tcc ttc act aac gtc act gtg gcc atg atc att gcc ttc tcc ttt agc tgg aag ctg agc ctg gtc atc ttg tgc ttc ttc ccc ttc ttg get tta tca gga gcc aca cag acC AGG atg ttg aca gga ttt gcc tct CGA gat aag cag gcc ctg gag atg gtg gga cag att aca aat gaa gcc ctc agt aac $\overline{\text { atc }}$ cgc act gtt get gga att gga $\overline{\text { aag }}$ gag $\overline{A G G}$ cgg ttc att gaa gca ctt gag act gag ctg gag aag ccc ttc aag aca gcc att cag aaa gcc aat att tac gga ttc tgc ttt gcc ttt gcc cag tgc atc atg ttt att gcg aat tct gct tcc tac AGA tat gga ggt tac tta atc tcc aat gag ggg ctc cat ttc agc tat gtg ttc AGG gtg atc tct gca gtt gta ctg agt gca aca gct ctt gga AGA gcc ttc tct tac acc cca agt tat gca aaa gct aaa $\overline{\text { ATA }}$ tca gct gca cgc ttt ttt caa ctg ctg gac CGA caa CCC cca atc agt gta tac aat act gca ggt gaa aaa tgg gac aac ttc cag ggg aag att gat ttt gtt gat tgt $\overline{\mathrm{aaa}}$ tt $\overline{\mathrm{aca}}$ tat cct tct $\mathrm{CGA}$ cct gac tcg caa gtt ctg aat ggt ctc tca gtg tcg att agt cca ggg cag aca ctg gcg ttt gtt ggg agc agt gga tgt ggc aaa agc act agc att cag ctg ttg gaa cgt ttc tat gat cct gat caa ggg aag gtg atg ATA gat ggt cat gac agc aaa aaa gta aat gtc cag ttc ctc cgc tca aac att gga att gtt tcc cag gaa cca gtg ttg ttt gcc tgt agc ATA atg gac aat atc aag tat gga gac aac acc aaa gaa att ccc atg gaa AGA gtc ATA gca gct gca aaa cag gct cag $\overline{\text { ctg }}$ cat gat ttt gtc atg tca ctc cca gag aaa tat gaa act aac gtt ggg $\overline{\text { tcc }}$ cag $\overline{g g g}$ tct caa ctc tct $\underline{A G A}$ ggg gag aaa caa cgc att gct att get cgg gcc att gta CGA gat cct aaa atc ttg CTA CTA gat gaa gcc act tct $\overline{g c c}$ tta gac aca gaa agt gaa aag acg gtg cag gtt gct CTA $\overline{\text { gac }}$ aaa gcc AGA gag ggt $\overline{c g g} \overline{\text { acc }}$ tgc att gtc att gcc cat $\mathrm{cgc}$ ttg tcc acc atc cag aac gcg gat atc att gct gtc atg gca cag ggg gtg gtg att gaa aag ggg acc cat gaa gaa ctg atg gec caa aaa gga gcc tac tac aa CTA gtc acc act gga tcc cCC atc agt tga

Figure 3: Representation the rare codon of Arg, Leu, Ile, and Pro in the ABCB11 gene. These residues display in red, blue, green, orange, and red, respectively.

are responsible for PFIC2 disease were presented previously [1]. In Figure 5, a large number of these mutations were highlighted in red color. As seen, these mutations were scattered throughout the nucleotide sequence of $\mathrm{ABCB} 11$ gene. In the following, those rare codons which were associated with mutations in $\mathrm{ABCB} 11$ and responsible for PFIC2 disease were focused. In this figure, we show some of these mutations in relation to rare codons.

Some of these mutations overlap rare codons and some of these mutations overlap the change of codons resulted in the production of new rare codons. After their comparison, these overlapping rare codons and new rare codons were structurally studied precisely. These rare codons identified in this study are located in different locations of BSEP protein structure (Table 2).

Analyzing the molecular model of BSEP showed that $\mathrm{Arg}^{415}$ residue (a residue with rare codon), forms a hydrogen bond with $\mathrm{Gln}^{414}$ (Figure 6). But, with mutation of $\mathrm{Arg}^{415}$ residue to Gln, this hydrogen bond was disrupted. The significance of this change is that this mutation caused BRIC disorder in these patients [25] (Figure 6). Furthermore, analyzing the 3D model of ATP8B1 structure in PIC server 


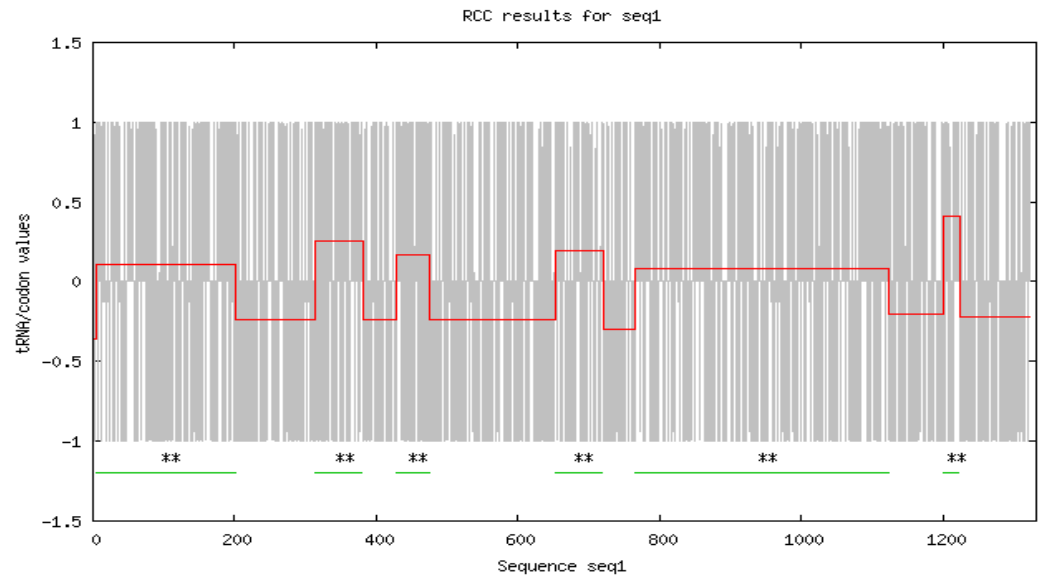

RCCs: slowly translating regions. RCCs correspond to positive averase values.

Transformed scale MSS average
RCC range

A

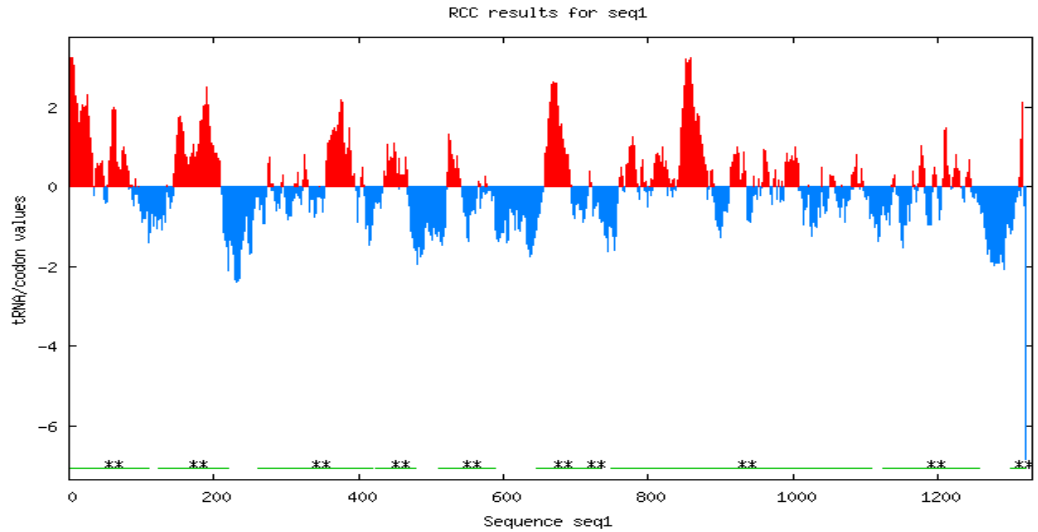

RCCs: slowly translating resions.

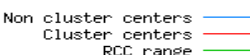

B

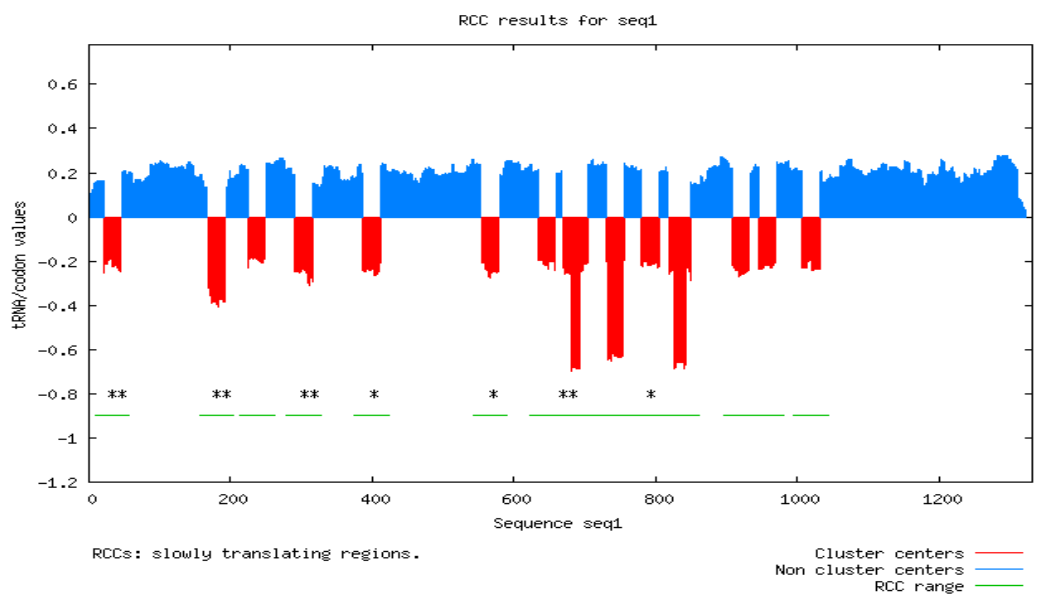

C

Figure 4: The representation of RCCs location in ABCB11 gene using MSS algorithm (A), minmax algorithm (B), and sliding window method (C). 
atg tct gac tca gta att ctt $\underline{\text { CGA }}$ agt ATA aag aaa ttt gga gag gag aat gat ggt ttt gag tca gat aaa tca tat aat aat gat aag aaa tca $\overline{A G G}$ tta $\overline{c a a}$ gat $\overline{g a g}$ aag aaa ggt gat ggc gtt $\overline{A G A}$ gtt ggc ttc ttt caa ttg ttt cgg ttt tct tca tca act gac att $\overline{\mathrm{tgg}} \mathrm{ctg}$ atg ttt gtg gga agt ttg tgt gca ttt $\overline{c t c}$ cat gga ATA gcc cag cca ggc gtg CTA ctc att ttt ggc aca atg aca gat gtt ttt att gac tac gac gtt gag tta caa gaa ctc $\overline{c a g}$ att cca gga aaa gca tgt gtg aat aac acc att gta tgg act aac agt tcc ctc aac cag aac atg aca aat gga aca cgt tgt ggg ttg ctg aac atc gag agc gaa atg atc aaa ttt gcc agt tac tat gct gga att gct gtc gca gta ctt atc aca gga tat att caa ATA tgc ttt tgg gtc att gcc gca gct cgt cag ATA cag aaa atg AGA aaa ttt tac ttt AGG AGA ATA atg AGA atg gaa ATA ggg tgg ttt gac tgc aat tca gtg ggg gag ctg aat aca AGA ttc tct gat gat att aat aa atc aat gat gcc ATA gct gac caa atg gcc ctt ttc att cag cgc atg acc tcg acc atc tgt ggt ttc ctg ttg gga ttt ttc AGG ggt tgg aaa ctg acc ttg gtt att att tct gtc agc cct ctc att ggg att gga gca gcc acc att ggt ctg agt gtg tcc aag ttt acg gac tat gac ctg aag gcc tat gcc aaa gca ggg gtg gtg gct gat gaa gtc att tca tca atg AGA aca gtg gct gct ttt ggt ggt gac aaa AGA gag gtt gaa AGG tat gag aaa aat ctt gtg ttc gcC cag cgt tgg gga att AGA aaa gga ATA gtg atg gga ttc ttt act gga ttc gtg $\overline{\operatorname{tgg}}$ tgt ctc atc ttt ttg tgt tat gca ctg gcc ttc tgg tac ggc tcc aca ctt gtc ctg gat gaa gga gaa tat aca cca gga acc ctt gtc cag att ttc ctc agt gtc ATA gta gga gct tta aat ctt ggc aat gcc tct cct tgt ttg gaa gcc ttt gca act gga cgt gca gca gcc acc agc att ttt gag aca ATA gac AGG aaa CCC atc att gac tgc

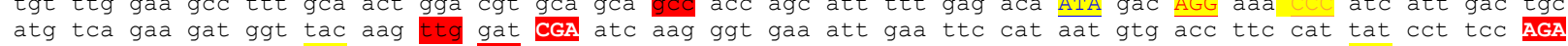
cca gag gtg aag att $\underline{\text { CTA }}$ aat gac ctc aac atg gtc att aaa cca ggg gaa atg aca gct ctg gta gga ccc agt gga get gga aad agt aca gca ctg caa ctc att cag CGA ttc tat gac CCC tgt gaa gga atg gtg acc gtg gat ggc cat gac att cgc tct ctt aac att cag tgg ctt AGA gat cag att ggg ATA gtg gag caa gag cca gtt ctg ttc tct acc acc att gca gaa aat att cgc tat ggc AGA gaa gat gca aca atg gaa gac ATA gtc caa gct gcc aag gag gcc aat gcc tac aac ttc atc atg gac ctg cca cag caa ttt gac acc ctt gtt gga gaa gga gga ggc cag atg agt ggt gge cag aaa caa AGG gta gcc atg gtg caa gaa gtg ctg agt aag att cag cat ggg cac aca atc att tca gtt gct cat cgc ttg tct acg gtc AGA gct gca gat acc atc att ggt tt gaa cat ggc act gca gtg gaa AGA ggg acc cat gaa gaa tta ctg gaa AGG aaa ggt gtt tac ttc act CTA gtg act ttg caa agc cag gga aat caa gct $\overline{c t}$ aat gaa gag gac ATA aag gat gca act gaa gat gac atg ctt gcg $\overline{\mathrm{AGG}}$ acc ttt agc $\overline{A G A}$ ggg agc tac cag gat agt tta $\mathrm{AGG}$ gct tcc atc $\overline{\mathrm{cgg}} \mathrm{caa}$ cgc tcc aag tct cag ctt tct tac ctg $\overline{g t g}$ cac gaa cct $\overline{c c a}$ tta gct gtt gta gat cat aag tct acc tat gaa gaa gat AGA aag gac aag gac att cct gtg cag gaa gaa gtt gaa cct gcc cca gtt AGG AGG att ctg aaa ttc agt gct cca gaa tgg ccc tac atg ctg gta ggg tct gtg ggt gca gct gtg aac ggg aca gtc aca $\overline{c c c}$ ttg tat gcc ttt tta ttc agc cag att ctt ggg act ttt tca att cct gat aaa gag gaa caa AGG tca cag atc aat ggt gtg tgc CTA ctt ttt gta gca atg ggc tgt gta tct ctt ttc acc caa ttt CTA cag gga tat $\overline{\text { gCC }}$ ttt gct aaa tct ggg gag ctc CTA aca aaa AGG CTA cgt aaa ttt ggt ttc AGG gca atg ctg ggg $\overline{c a a}$ gat att gcc tgg ttt gat gac ctc $\overline{A G A}$ aat agc $\overline{c c t}$ gga gca $\overline{\text { ttg }} \overline{\text { aca }}$ aca $\overline{A G A}$ ctt gct aca gat gct tcc caa gtt caa ggg gct gcc ggc tct cag atc ggg atg ATA gtc aat tcc ttc act aac gtc act gtg gcc atg atc att gcc ttc tcc ttt agc tgg aag ctg agc ctg gtc atc ttg tgc ttc ttc ccc ttc ttg gct tta tca gga gcc aca cag acc AGG atg ttg aca gga ttt gcc tct CGA gat aag cag gcc ctg gag atg gtg gga cag att aca aat gaa gcc ctc agt aac atc cge act gtt gct gga att gga aag gag AGG cgg ttc att gaa gca ctt gag act gag ctg gag aag cce ttc aag aac atc cgc act gtt gct gga att gga aag gag AGG cgg ttc att gaa gca ctt gag act gag ctg gag aag cce ttc aag
aca gcc att cag aaa gcc aat att tac gga ttc tgc ttt gcc ttt gcc cag tgc atc atg tet att gcg aat tct gct tcc tac AGA tat gga ggt tac tta atc tcc aat gag ggg ctc cat ttc agc tat gtg ttc AGG gtg atc tct gca gtt gta ctg agt gca aca get ctt gga AGA gcc ttc tct tac acc cca agt tat gca aaa gct aaa ATA tca gct gca cac ttt ttt caa ctg ctg gac CGA caa CCC CCa atc agt gta tac aat act gca ggt gaa aaa tgg gac aac ttc cag ggg aag att gat ttt gtt gat tgt aaa ttt aca tat cct tct CGA cct gac tcg caa gtt ctg aat ggt ctc tca gtg tcg att agt cca ggg cag aca ctg gcg ttt gtt ggg agc agt gga tgt ggc aaa agc act agc att cag ctg ttg gaa cgt ttc tat gat cct gat caa ggg aag gtg atg ATA gat ggt cat gac agc aaa aaa gta aat gtc cag ttc ctc cgc tca aac att gga att gtt tcc cag gaa cca gtg ttg ttt gCC tgt agc ATA atg gac aat atc aag tat gga gac aac acc aaa gaa att ccc atg gaa AGA gtc ATA gca gct gca aaa cag gct cag ctg cat gat ttt gtc atg tca ctc cca gag aaa tat gaa act aac gtt ggg tcc cag $\overline{g g g}$ tct caa ctc tct AGA ggg gag aaa caa cgc att gct att get cgg gcc att gta CGA gat cct aaa atc ttg CTA

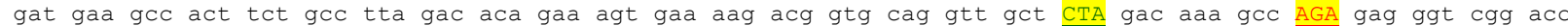
tgc att gtc att gcc cat cgc ttg tcc acc atc cag aac gcg gat atc att gct gtc atg gca cag ggg gtg gtg att gaa aag ggg acc cat gaa gaa ctg atg gec caa aaa gga gcc tac tac aaa CTA gtc acc act gga tcc cce atc agt tga

Figure 5: Schematic representation of the codon usage of $A B C B 11$ gene and position of rare codons and mutations, highlighted in yellow and red, respectively.

showed the interaction of these residues with other residues.

Another missense mutation (1295 G>C) was also detected resulting in PFIC (Figure 7) [26]. In this mutation, the codon sequence of $\mathrm{Arg}^{432}$ (AGA as rare codon) changed to $\mathrm{Thr}^{432}$ (ACA). These original and substituted residues constitute hydrogen bonds with $\mathrm{Ser}^{431}$ as shown in Figure 7B. The significance of this change is that $\operatorname{Arg}^{432}$ is located in the interior space of BSEP protein channel, and this mutation caused BRIC disorder [26] (Figure 7).

Analyzing the ATP8B13D model showed that $\mathrm{Arg}^{470}$ residue (a residue with rare codon), forms a hydrogen bond with $\mathrm{Leu}^{467}, \mathrm{Ile}^{468}$, $\mathrm{Ala}^{472}, \mathrm{Asp}^{473}, \mathrm{Asp}^{485}$ and $\mathrm{Gln}^{466}$ (Figure 8). But, with mutation of $\mathrm{Arg}^{470}$ residue to Gln, some of these hydrogen bonds were disrupted. This mutation also caused BRIC disorder in patients with this mutation in the ABCB11 gene [27] (Figure 8).

Another mutation (3724 C>A) was also detected that resulted to PFIC (Figure 9A) [27]. In this mutation, the codon sequence of Leu ${ }^{1242}$ (CTA as rare codon) changed to $\mathrm{Ile}^{1242}$ (ATA). These original and substituted residues constitute the hydrogen bonds with $\mathrm{Ser}^{431}$ shown in Figure 9B Ile ${ }^{1273}$. 
Table 2: The 1-4 numbers are the position of rare codon in $A B C B 11$ gene that mutated to the non-rare codon and 5-12 numbers are the position of non-rare codons that mutated to the rare codon in the PFIC2 patients and analyzed.

\begin{tabular}{cccccc} 
Number & Exon & Nucleotide Change & Predicted Protein Effect & Location in Protein & Reference \\
\hline 1 & 12 & c. $1244 G>A$ & R415Q & NBF & {$[25]$} \\
\hline 2 & 12 & c. $1295 G>C$ & R432T & NBF & {$[26]$} \\
\hline 3 & 13 & c. $1409 G>A$ & R470Q & Adj WA & {$[27]$} \\
\hline 4 & 27 & c.3724C>A & L1242I & WB & {$[27]$} \\
\hline 5 & 11 & c. $1168 G>C$ & A390P & NBF & {$[27]$} \\
\hline 6 & 15 & c.1779T>A & S593R & NBF1 & {$[28]$} \\
\hline 7 & 13 & c. $1388 C>T$ & T463I & WA & {$[27]$} \\
\hline 8 & 18 & c. $2130 T>C$ & P710P & IC3 & {$[29]$} \\
\hline 9 & $20-21$ & c. $2576 C>C$ & T859R & IC4 & {$[27]$} \\
\hline 10 & 22 & c. $2776 G>C$ & A926P & IC5 & {$[30]$} \\
\hline 11 & 23 & c. $2944 G>A$ & G982R & TM11 & {$[31]$} \\
\hline 12 & 25 & c. $3346 G>C$ & G1116R & WA &
\end{tabular}

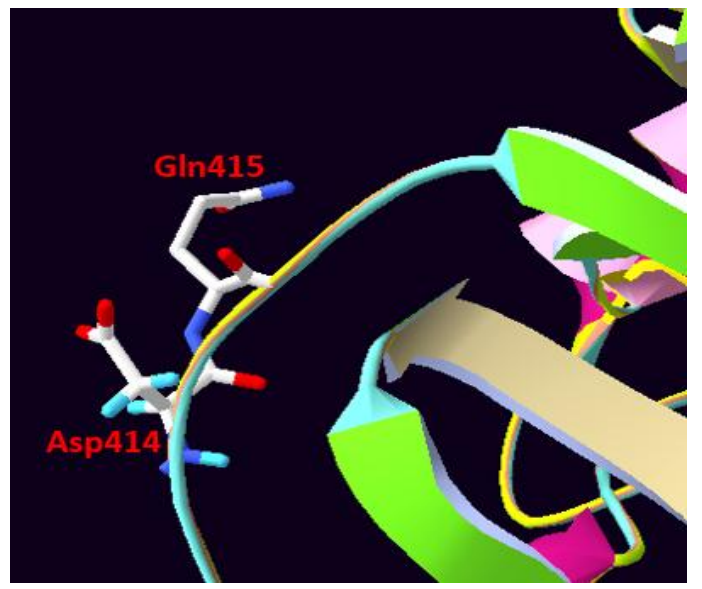

A

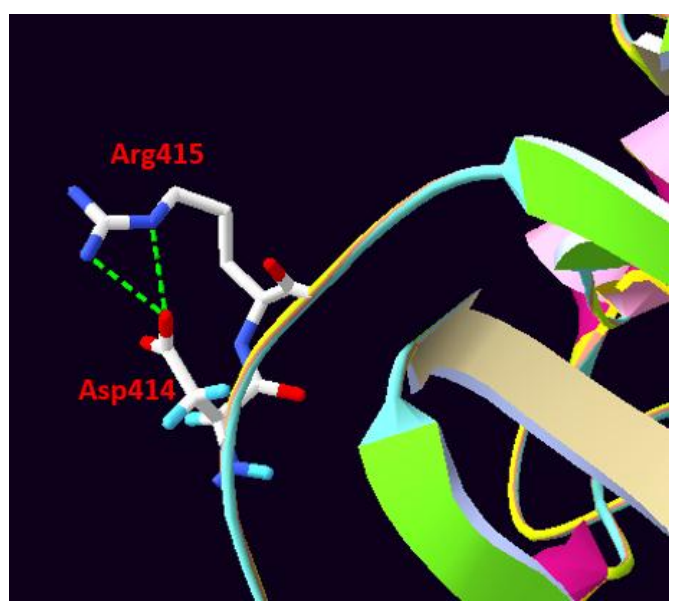

B

Figure 6: A) the ribbon diagram of BSEP protein, with location of $\mathrm{Arg}^{415}$ residue (rare codon). The $\operatorname{Arg}^{415}$ residue forms the hydrogen bond with $\mathrm{Gln}^{414}$ B) mutation $\operatorname{Arg}^{415}$ to $\operatorname{Gl}^{415}$.

\section{Discussion}

Three types of PFIC which are referred to autosomal-recessive liver disorders are related to mutations in hepatocellular transportsystem genes [28-33]. Mutations in ABCB11 gene have a variety in phenotype of autosomal recessive cholestasis liver diseases [1]
Liver disease in BSEP deficiency attributes to intrahepatocytic accumulation of toxic bile salts and failed the secretion of toxic bile salts [29]. Our comprehensive evaluation of these ABCB11 mutations show that all of these mutations that are resulted in cholestasis liver diseases are scattered throughout the gene and 


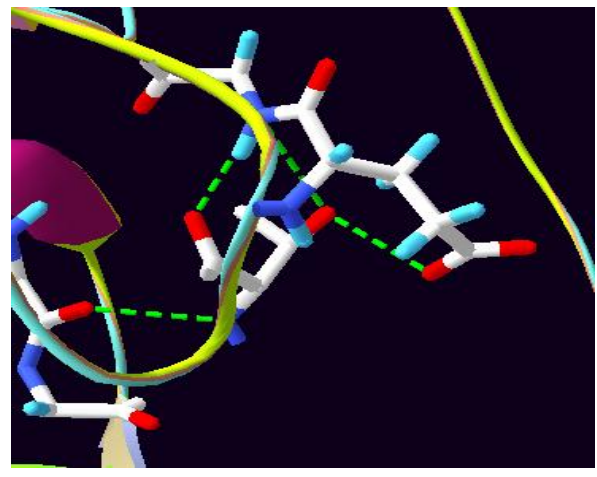

A

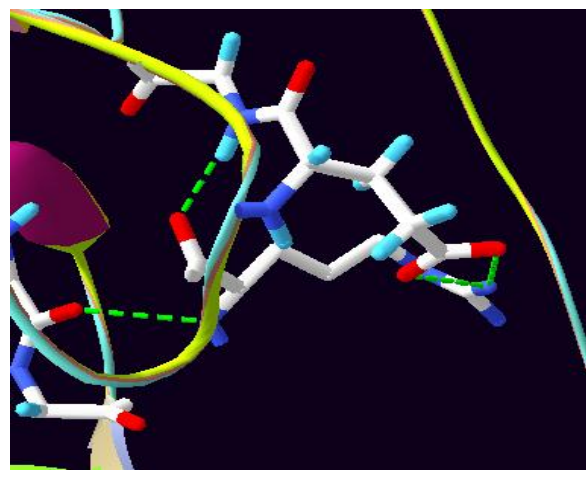

B

Figure 7: A) the ribbon representation of BSEP protein, with location of $\operatorname{Arg}^{432}$ residue (rare codon). B) Mutation of $\operatorname{Arg}^{432}$ to $\mathrm{Thr}^{432}$.

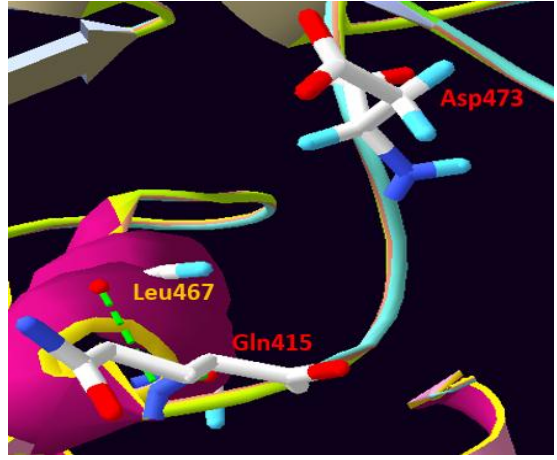

A

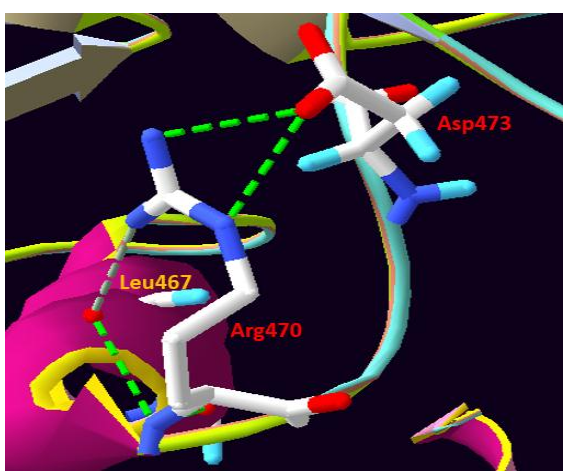

B

Figure 8: A) the ribbon diagram of BSEP protein, with location of $\operatorname{Arg}^{470}$ residue (rare codon). The $\operatorname{Arg}^{470}$ residue forms the hydrogen bond with $\mathrm{Asp}^{473} \mathrm{~B}$ ) mutation $\operatorname{Arg}^{470}$ to $\mathrm{Gln}^{470}$.

are not concentrated in special regions of this gene. This tells that the protein structure and function of BSEP has a very high sensitivity to the mutations and structural changes. This shows that any mutation in this structure can have destructive effects on the structure and function of BSEP. Re-evaluation of this mutation can provide a new approach in the study of these patients and design of new drugs. In this regard, considering the hidden information as "rare" codons that are infrequently used by cells and the specific roles of these rare codons in the proper folding of proteins is critical.

We have previously conducted the identification of detection of rare codons and molecular modelling of some proteins in our lab and have a good experience in these techniques [34-39]. For better evaluation of ABCB11 gene in this study, the detecting and studying of rare codons were conducted. In the following, the relation of some ABCB11 mutation with rare codons was studied. For the detection of rare codons, the following web server was used. For Pfam detection, the UniProt database identified two Pfams for ABCB11 as PF00664 (ABC_mem- 


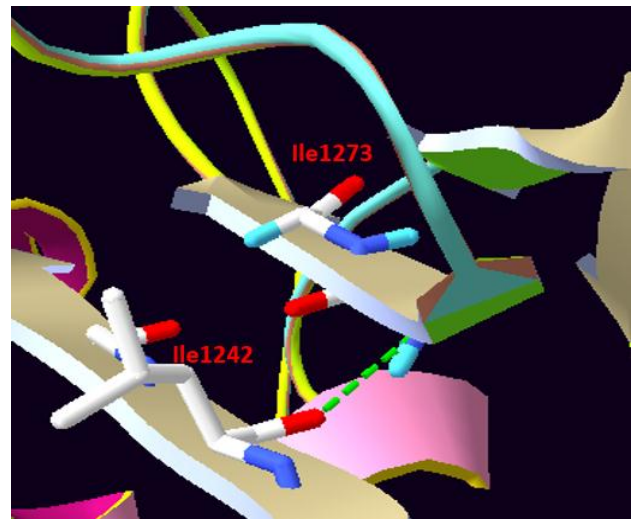

A

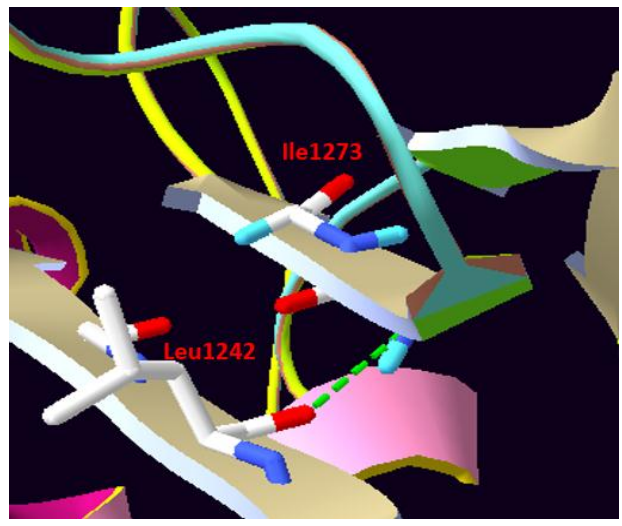

$\mathrm{B}$

Figure 9: A) The ribbon diagram of BSEP protein, with location of Leu ${ }^{1242}$ residue (rare codon). B) Mutation of Leu $u^{1242}$ to $\mathrm{ll}^{1242}$.

brane. 2 hits) and PF00005 (ABC_tran. 2 hits). These Pfams were analyzed in the Sherlocc program that identified no rare codon clusters in the ABCB11 gene. In the following, this sequence nucleotide of this gene was analyzed in the ATGme web server that detected the 69 rare codon and 10 highly rare codons that may have a critical role in proper folding of protein chain. In addition, this gene was analyzed in the RaCC server detecting 48 rare codons for Arg, 18 rare codons for Ile, 11 rare codon for Leu and 11 rare codons for Pro. Finally, using LaTcOm web tool, the RCCs of this gene were detected. Results showed a large number of RCCs in the ABCB11 gene in these three algorithms.

The overall evaluation of rare codons of ABCB11 gene showed a large number of rare codons and rare codon clusters. This connotes that the protein structure of ABCB11 has important hidden features that need to guarantee the proper folding of this protein. For this reason, a large number of rare codons slowly had done the overall folding rate of this pump so that the final protein has a correct structure and function. However, these results show a large number of the rare codons of Arg and with large number of non-covalent hydrogen bonds play a special role in the correct folding of ABCB11. Finally, we focus on some rare codons related to PFIC2. In this regard, 3D molecular modelling of ABCB11 was conducted in I-TSSAR Web Server.

The precise analysis reveals that four rare codons were mutated in PFIC2 disease. These four rare codons are distributed in different regions in the structure of BSEP protein (Figure $10)$.

$\operatorname{Arg}^{859}$ (rare codon). E) The ribbon diagram of BSEP protein, with location of Gly ${ }^{982}$ residue. F) Mutation Gly ${ }^{982}$ to Arg $^{982}$ (rare codon). G) The ribbon diagram of BSEP protein, with location of Gly ${ }^{1116}$ residue. H) Mutation Gly ${ }^{1116}$ to $\operatorname{Arg}^{1116}$ (rare codon).

Structural analysis demonstrates that these rare codons form some hydrogen bonds with other residues disrupted with mutation in PFIC2 patients. This mutation with the disruption of these hydrogen bonds or change in the protein folding rate affects the protein folding that may disrupt the proper structure and function of $A B C B 11$. It shows the critical role of these residues in the process of protein folding. However, other hypotheses should be considered too.

Table 2 shows new rare codons which are 


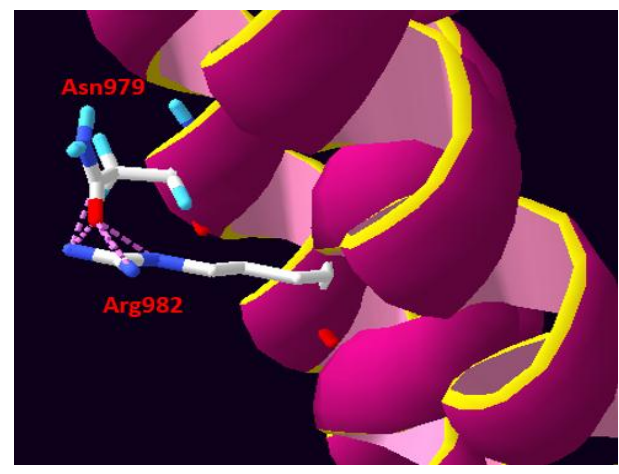

$\mathrm{E}$

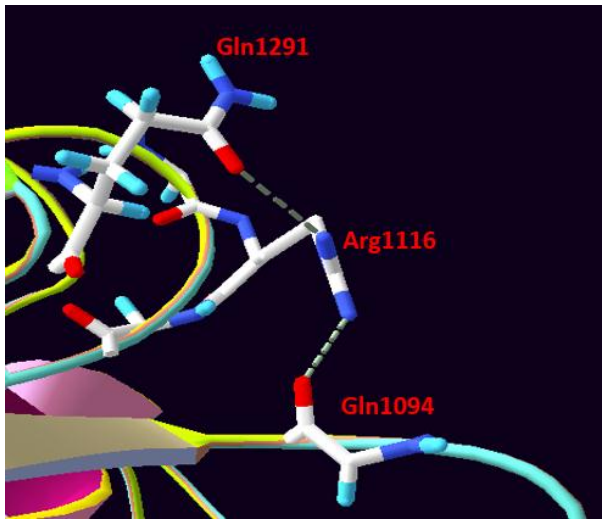

G

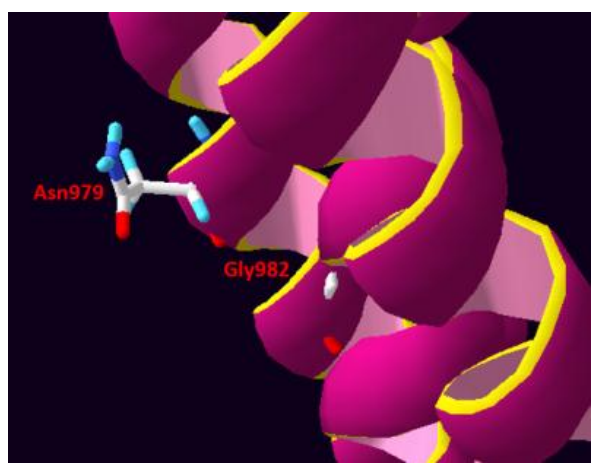

$\mathrm{F}$

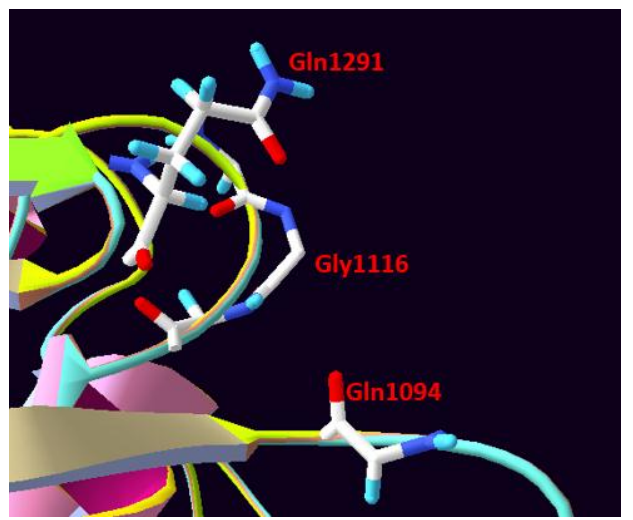

$\mathrm{H}$

Figure 10: A) The ribbon diagram of BSEP protein, with location of Ser ${ }^{539}$ residue. B) mutation $\operatorname{Ser}^{539}$ to $\operatorname{Arg}^{539}$ (rare codon). C) The ribbon diagram of BSEP protein, with location of Thr ${ }^{859}$ residue. D) mutation $\mathrm{Thr}^{859}$ to $\operatorname{Arg}^{859}$ (rare codon). E) The ribbon diagram of BSEP protein, with location of Gly ${ }^{982}$ residue. F) Mutation Gly ${ }^{982}$ to $\operatorname{Arg}^{982}$ (rare codon). G) The ribbon diagram of BSEP protein, with location of Gly ${ }^{1116}$ residue. H) Mutation $\mathrm{Gly}^{1116}$ to $\operatorname{Arg}^{1116}$ (rare codon).

caused by some mutations of PFIC2 patients. In these patients, non-rare codons were converted to rare codons that interfere with suitable protein folding rate. These mutations change the hydrogen bond network affecting the structure and function of BSEP protein. On the other hand, these mutations changed the protein folding rate interfering with correct protein folding. Besides this, these new residues have a different side chain in comparison with original residues that may create the structural repulsion interfering with proper folding and functional activity of BSEP. Final- ly, these mutations either by change of folding rate or by change of hydrogen interaction have a negative effect on the BSEP and result in PFIC2 disease.

\section{Conclusion}

Meanwhile, some in-vivo and in-silico evidence as molecular docking and evaluating these mutations is needed for our theoretical study confirmation. Our data showed that rare codon positions might have an essential role in folding and activity of BSEP. This study may also provide new insights into drug de- 
sign for the treatment of PFIC2, in the future. Acknowledgment

The study was performed as a $\mathrm{PhD}$ thesis in shiraz University of Medical Sciences with grant No. of 93-7346.

Data Sharing Statement: All data were extracted from database of clinics and hospitals affiliated to Shiraz University of Medical Sciences.

\section{Conflict of Interest}

There is no conflict of interest for this study.

\section{References}

1. Byrne JA, Strautnieks SS, Ihrke G, Pagani F, Knisely AS, Linton KJ, et al. Missense mutations and single nucleotide polymorphisms in ABCB11 impair bile salt export pump processing and function or disrupt pre-messenger RNA splicing. Hepatology. 2009;49:553-67. doi.org/10.1002/ hep.22683. PubMed PMID: 19101985.

2. Jacquemin E. Progressive familial intrahepatic cholestasis. Clinics and research in hepatology and gastroenterology. 2012;36:S26-S35. doi. org/10.1016/S2210-7401(12)70018-9.

3. Sira AM, Sira MM. Progressive familial intrahepatic cholestasis: INTECH Open Access Publisher; 2013.

4. Reichel C, Meier-Abt PJ. Cholestatic liver diseases. Ther Umsch. 1997;54:639-44. PubMed PMID: 9454366.

5. Engelmann G, Wenning D, Herebian D, Sander 0, Droge C, Kluge S, et al. Two Case Reports of Successful Treatment of Cholestasis With Steroids in Patients With PFIC-2. Pediatrics. 2015;135:e132632. doi.org/10.1542/peds.2014-2376. PubMed PMID: 25847799.

6. Fathy M, Kamal M, Al-Sharkawy M, Al-Karaksy $\mathrm{H}$, Hassan N. Molecular characterization of exons 6,8 and 9 of ABCB4 gene in children with Progressive Familial Intrahepatic Cholestasis type 3 . Biomarkers. 2016;21:573-7. doi.org/10.3109/135 4750X.2016.1166264. PubMed PMID: 27075526.

7. Carlton VE, Knisely AS, Freimer NB. Mapping of a locus for progressive familial intrahepatic cholestasis (Byler disease) to 18q21-q22, the benign recurrent intrahepatic cholestasis region. Hum Mol Genet. 1995;4:1049-53. doi.org/10.1093/ hmg/4.6.1049. PubMed PMID: 7655458.

8. Dröge $C$, Kluge $S$, Häussinger $D$, Kubitz R, Keitel $V$. Sequencing of ATP8B1, ABCB11 and ABCB4 revealed 135 genetic variants in 374 unrelated patients with suspected intrahepatic cholestasis. Zeitschrift für Gastroenterologie. 2015;53:A3_27.

9. Kane JF. Effects of rare codon clusters on highlevel expression of heterologous proteins in Escherichia coli. Curr Opin Biotechnol. 1995;6:494500. doi.org/10.1016/0958-1669(95)80082-4. PubMed PMID: 7579660.

10. Chartier M, Gaudreault F, Najmanovich R. Largescale analysis of conserved rare codon clusters suggests an involvement in co-translational molecular recognition events. Bioinformatics. 2012;28:1438-45. doi.org/10.1093/bioinformatics/bts149. PubMed PMID: 22467916. PubMed PMCID: 3465090.

11. Giovannoni I, Callea F, Bellacchio E, Torre G, De Ville De Goyet J, Francalanci P. Genetics and Molecular Modeling of New Mutations of Familial Intrahepatic Cholestasis in a Single Italian Center. PLOS One. 2015;10:e0145021. doi.org/10.1371/ journal.pone.0145021. PubMed PMID: 26678486. PubMed PMCID: 4683058.

12. Zhang Y. I-TASSER server for protein 3D structure prediction. BMC Bioinformatics. 2008;9:40. doi.org/10.1186/1471-2105-9-40. PubMed PMID: 18215316. PubMed PMCID: 2245901.

13. Thanaraj TA, Argos P. Protein secondary structural types are differentially coded on messenger RNA. Protein Sci. 1996;5:1973-83. doi. org/10.1002/pro.5560051003. PubMed PMID: 8897597. PubMed PMCID: 2143259.

14. Theodosiou A, Promponas VJ. LaTcOm: a web server for visualizing rare codon clusters in coding sequences. Bioinformatics. 2012;28:591-2. doi.org/10.1093/bioinformatics/btr706. PubMed PMID: 22199385.

15. Daniel E, Onwukwe GU, Wierenga RK, Quaggin SE, Vainio SJ, Krause M. ATGme: Open-source web application for rare codon identification and custom DNA sequence optimization. BMC Bioinformatics. 2015;16:303. doi.org/10.1186/s12859015-0743-5. PubMed PMID: 26391121. PubMed PMCID: 4578782.

16. Wu S, Zhang Y. LOMETS: a local meta-threadingserver for protein structure prediction. Nucleic Acids Res. 2007;35:3375-82. doi.org/10.1093/ nar/gkm251.

17. DeLano WL. The PyMOL molecular graphics system. San Carlos: PyMOL; 2002.

18. Kaplan W, Littlejohn TG. Swiss-PDB Viewer (Deep View). Brief Bioinform. 2001;2:195-7. doi.org/10.1093/bib/2.2.195. PubMed PMID: 11465736. 
19. Tina K, Bhadra R, Srinivasan N. PIC: Protein Interactions Calculator. Nucleic Acids Res. 2007;W473-6.

20. Vriend G. WHAT IF: a molecular modeling and drug design program. J Mol Graph. 1990;8:52-6, 29. PubMed PMID: 2268628.

21. Gruber A, Durham A, Huynh C, del Portillo H. Bioinformatics in tropical disease research: a practical and case-study approach. Bethesda (MD): National Library of Medicine (US), NCBI; 2008.

22. Gasteiger E, Hoogland C, Gattiker A, Duvaud Se, Wilkins MR, Appel RD, et al. Protein identification and analysis tools on the ExPASy server: Springer; 2005.

23. Sonnhammer EL, Eddy SR, Durbin R. Pfam: a comprehensive database of protein domain families based on seed alignments. Proteins. 1997;28:405-20. doi.org/10.1002/(SICI)10970134(199707)28:3<405::AID-PROT10>3.0.C0;2L. PubMed PMID: 9223186.

24. Dong $H$, Nilsson L, Kurland CG. Co-variation of tRNA abundance and codon usage in Escherichia coli at different growth rates. $J$ Mol Biol. 1996;260:649-63. doi.org/10.1006/ jmbi.1996.0428. PubMed PMID: 8709146.

25. Pauli-Magnus C, Lang T, Meier $Y$, Zodan-Marin T, Jung $D$, Breymann $C$, et al. Sequence analysis of bile salt export pump (ABCB11) and multidrug resistance $p$-glycoprotein 3 (ABCB4, MDR3) in patients with intrahepatic cholestasis of pregnancy. Pharmacogenetics. 2004;14:91-102. doi.org/10.1097/00008571-200402000-00003. PubMed PMID: 15077010.

26. Noe J, Kullak-Ublick GA, Jochum W, Stieger B, Kerb R, Haberl M, et al. Impaired expression and function of the bile salt export pump due to three novel ABCB11 mutations in intrahepatic cholestasis. J Hepatol. 2005;43:536-43. doi.org/10.1016/j. jhep.2005.05.020. PubMed PMID: 16039748.

27. Strautnieks SS, Byrne JA, Pawlikowska L, Cebecauerova $D$, Rayner $A$, Dutton $L$, et al. Severe bile salt export pump deficiency: 82 different ABCB11 mutations in 109 families. Gastroenterology. 2008;134:1203-14. doi.org/10.1053/j.gastro.2008.01.038. PubMed PMID: 18395098.

28. Jansen PL, Strautnieks SS, Jacquemin E, Hadchouel M, Sokal EM, Hooiveld GJ, et al. Hepatocanalicular bile salt export pump deficiency in patients with progressive familial intrahepatic cholestasis. Gastroenterology. 1999;117:13709. doi.org/10.1016/S0016-5085(99)70287-8. PubMed PMID: 10579978.

29. Pauli-Magnus C, Kerb R, Fattinger K, Lang T,
Anwald B, Kullak-Ublick GA, et al. BSEP and MDR3 haplotype structure in healthy Caucasians, primary biliary cirrhosis and primary sclerosing cholangitis. Hepatology. 2004;39:779-91. doi.org/10.1002/hep.20159. PubMed PMID: 14999697.

30. van Mil SW, van der Woerd WL, van der Brugge G, Sturm E, Jansen PL, Bull LN, et al. Benign recurrent intrahepatic cholestasis type 2 is caused by mutations in ABCB11. Gastroenterology. 2004;127:379-84. doi.org/10.1053/j.gastro.2004.04.065. PubMed PMID: 15300568.

31. Strautnieks SS, Bull LN, Knisely AS, Kocoshis SA, Dahl N, Arnell $\mathrm{H}$, et al. A gene encoding a liverspecific $A B C$ transporter is mutated in progressive familial intrahepatic cholestasis. Nat Genet. 1998;20:233-8. doi.org/10.1038/3034. PubMed PMID: 9806540.

32. Erlinger S, Arias IM, Dhumeaux D. Inherited disorders of bilirubin transport and conjugation: new insights into molecular mechanisms and consequences. Gastroenterology. 2014;146:1625-38. doi.org/10.1053/j.gastro.2014.03.047. PubMed PMID: 24704527.

33. Nicolaou M, Andress EJ, Zolnerciks JK, Dixon PH, Williamson C, Linton KJ. Canalicular ABC transporters and liver disease. J Pathol. 2012;226:30015. doi.org/10.1002/path.3019. PubMed PMID: 21984474.

34. Bina S, Shenavar F, Khodadad M, Haghshenas MR, Mortazavi M, Fattahi MR, et al. Impact of RGD Peptide Tethering to IL24/mda-7 (Melanoma Differentiation Associated Gene-7) on Apoptosis Induction in Hepatocellular Carcinoma Cells. Asian Pac J Cancer Prev. 2015;16:6073-80. doi. org/10.7314/APJCP.2015.16.14.6073. PubMed PMID: 26320498.

35. Fattahi M, Malekpour A, Mortazavi M, Safarpour A, Naseri N. The characteristics of rare codon clusters in the genome and proteins of hepatitis C virus; a bioinformatics look. Middle East J Dig Dis. 2014;6:214-27. PubMed PMID: 25349685. PubMed PMCID: 4208930.

36. Mortazavi M, Hosseinkhani S. Surface charge modification increases firefly luciferase rigidity without alteration in bioluminescence spectra. Enzyme Microb Technol. 2017;96:47-59. doi. org/10.1016/j.enzmictec.2016.09.014. PubMed PMID: 27871385.

37. Mortazavi M, Hosseinkhani S. Design of thermostable luciferases through arginine saturation in solvent-exposed loops. Protein Eng Des Sel. 2011;24:893-903. doi.org/10.1093/protein/ 
gzr051. PubMed PMID: 22068960.

38. Mortazavi M, Zarenezhad M, Alavian SM, Gholamzadeh S, Malekpour A, Ghorbani M, et al. Bioinformatic Analysis of Codon Usage and Phylogenetic Relationships in Different Genotypes of the Hepatitis C Virus. Hepat Mon. 2016;16:e39196. doi.org/10.5812/hepatmon.39196. PubMed PMID: 27882066. PubMed PMCID: 5111459.
39. Mortazavi M, Zarenezhad M, Gholamzadeh S, Alavian SM, Ghorbani M, Dehghani R, et al. Bioinformatic Identification of Rare Codon Clusters (RCCs) in HBV Genome and Evaluation of RCCs in Proteins Structure of Hepatitis B Virus. Hepat Mon. 2016;16:e39909 . doi.org/10.5812/hepatmon.39909. PubMed PMID: 27882067. PubMed PMCID: 5116127. 